\title{
Gestion de la qualité: transmettre un savoir pour promouvoir la qualité
}

\begin{abstract}
Situation initiale
Le contrôle régulier de la qualité, sa garantie et son amélioration, sont des exigences auxquelles les prestations médicales doivent elles aussi satisfaire. La FMH, qui est l'organisation faîtière des médecins suisses, soutient ces exigences. Elle a donc élaboré un document de base intitulé «Gestion de la qualité et certification de la prestation médicale: coûts et utilité» (cf. article ci-avant). Ce document montre l'étendue des possibilités de garantir et d'améliorer la qualité des prestations médicales. Il présente, d'une part, des systèmes nationaux et internationaux reconnus de gestion de la qualité pour le secteur ambulatoire et hospitalier. Il expose, d'autre part, les possibilités offertes aux médecins de développer la qualité de manière individuelle, en fonction de leurs besoins.
\end{abstract}

\section{Transmettre un savoir en matière de qualité}

Pour la FMH, l'application de mesures visant à améliorer la qualité est de première importance. Elle encourage les processus et mesures suscitant un apprentissage personnel continu de gestion de la qualité. La FMH, en particulier son département Données, démographie et qualité, prépare des bases en matière d'assurance et de développement de la qualité des prestations médicales. Pour ce faire, elle recueille les expériences faites avec différents systèmes de gestion de la qualité et instruments garantissant la qualité, transmet

Garantir et améliorer la qualité fait partie des tâches essentielles du médecin.

- La FMH s'engage pour I'assurance et l'amélioration de la qualité; elle recommande à ses membres d'appliquer les mesures adéquates.

- L'éventail des mesures visant à garantir et à améliorer la qualité des prestations médicales est étendu; il va de mesures individuelles à l'application de systèmes internationaux de gestion de la qualité.

- Il existe en Suisse différentes initiatives pour gérer la qualité. Dans ce cadre ont été développés entre autres des instruments et modèles standardisés, convaincants et faciles à appliquer.

- La FMH transmet à ses membres les connaissances nécessaires et leur fournit des conseils. les connaissances ainsi obtenues à ses membres et les conseille dans ce domaine.

\section{Etablir la transparence}

L'activité médicale doit pouvoir être évaluée quant à sa qualité et elle doit donc être rendue transparente. L'effet d'auto-apprentissage espéré implique toutefois aussi la confidentialité nécessaire. Les expériences faites par l'aéronautique montrent que les systèmes d'annonce d'erreurs fonctionnent uniquement si cette confidentialité est garantie. La transparence et la confidentialité doivent être soigneusement soupesées l'une par rapport à l'autre.

Pour établir la transparence, on peut recourir aux systèmes de gestion de la qualité développés par des institutions accréditées comme ISO, EFQM, EQUAM et sanaCERT. On peut aussi utiliser les instruments standardisés de gestion de la qualité qui sont à la disposition du corps médical en Suisse, tels qu'EPA et Quali-med-net. Des mesures individuelles peuvent également servir au développement de la qualité mais elles ne conviennent guère aux comparaisons.

L'attestation de la qualité ainsi que la transparence qui en résulte entraînent des coûts supplémentaires. La FMH est d'avis que ces coûts devraient être reconnus et pris en compte par des systèmes d'incitation appropriés; elle élabore les bases nécessaires à cette fin.

\section{Informer au sujet des certifications}

Pour la certification, on n'examine pas le «système apprenant» appliqué à la gestion de la qualité mais l'on vérifie si les exigences de qualité sont remplies. La certification en soi ne dit pas obligatoirement tout de la qualité des prestations médicales fournies. S'agissant de la transparence de la qualité, les certifications donnent toutefois l'occasion de procéder à des comparaisons et l'on peut envisager d'y recourir lorsqu'on applique des systèmes de gestion de la qualité. Elles sont possibles pour la plupart des systèmes standardisés. La FMH met à la disposition de ses membres des informations relatives aux certifications et les conseille volontiers. 